\title{
Erratum to: The Toxicological Effects in Brain of Mice Following Exposure to Cerium Chloride
}

\author{
Haiquan Zhao Z Zhe Cheng • Jie Cheng • Renping Hu • \\ Yi Che $\cdot$ Yaling Cui $\cdot$ Ling Wang $\cdot$ Fashui Hong
}

Published online: 22 May 2012

(C) Springer Science+Business Media, LLC 2012

Erratum to: Biol Trace Elem Res (2011) 144:872-884

DOI 10.1007/s12011-011-9045-6

The original version of this article unfortunately contained a mistake. The surnames of Haiquan Zhao and Jie Cheng were mispelled and are now corrected in this article. Instead of "Haiquan Zha" it should read "Haiquan Zhao" and "Jie Chen" should read as "Jie Cheng".

Haiquan Zhao, Zhe Cheng, and Jie Cheng contributed equally to this work.

The online version of the original article can be found at http://dx.doi.org/ 10.1007/s12011-011-9045-6.

H. Zhao $\cdot$ Z. Cheng $\cdot$ J. Cheng $\cdot$ R. Hu $\cdot$ Y. Che $\cdot$ Y. Cui $\cdot$

L. Wang $\cdot$ F. Hong $(\triangle)$

Medical College of Soochow University,

Suzhou 215123, People's Republic of China

e-mail: Hongfsh_cn@sina.com

H. Zhao

College of Life Sciences, Anhui Agriculture University,

Hefei 230036, People's Republic of China 